\title{
Preface
}

\section{Acute Kidney Injury in the Intensive Care Unit: Advances in the Identification, Classification, and Treatment of a Multifactorial Syndrome}

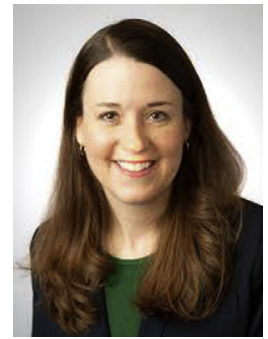

Dana Y. Fuhrman, DO, MS

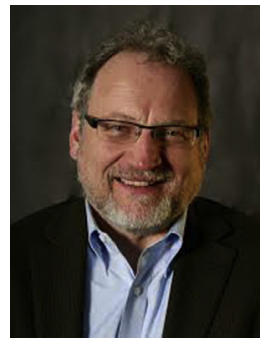

John A. Kellum MD, MCCM

\section{Editors}

Acute kidney injury (AKI) remains an epidemic in the intensive care unit (ICU), occurring in more than $50 \%$ of patients and with a mortality of $20 \%$ to $25 \% .{ }^{1}$ With the development of a standard for defining AKI with the Kidney Disease: Improving Global Outcomes (KDIGO) criteria in 2012, ${ }^{2}$ arguably no other area of study in critical care medicine has seen as rapid a rise in research efforts when compared with critical care nephrology. In 2020, there is no indication that these efforts are slowing, with still much work to be done.

A unified definition of AKI has allowed us to maintain consistency and provide a standard for defining AKI in research studies and in clinical practice. Study results in neonates, children, and adults have made it clear that we need to include oliguria when defining AKI. ${ }^{3-5}$ Given that worsening kidney function can occur over hours, refining our ability to predict changes in serum creatinine and urine output is crucial. The quest for the biomarkers to detect AKI early, determine progression and need for renal replacement therapy (RRT), and prediction of long-term outcomes continues. It has become increasingly clear that no single biomarker can fill these diverse roles, but instead, different biomarkers will be needed for different intended uses.

It is now evident that AKI is a syndrome with multiple causes. Recognizing that AKI "behaves" differently in different patient groups, investigators have focused their efforts on studying potentially vulnerable patients. The recent multicenter AWAKEN study has alerted us to the myriad of issues related to AKI in the neonatal period, 
including the need for RRT devices for our smallest patients. ${ }^{3}$ In addition, exciting recent advances in the pathophysiology of sepsis-associated AKI have shown us that $\mathrm{AKI}$ in the setting of systemic infection should be regarded as a "sepsis-defining event," occurring in the absence of signs of renal hypoperfusion. ${ }^{6}$ Importantly, research efforts dedicated to patients with cardiorenal syndrome, hepatorenal syndrome, and oncologic diseases have expanded greatly.

Recent advancements have improved our understanding of the management of AKI in the ICU. Satisfying our need for meticulous attention to fluid balance, new technologies to monitor fluid overload in the ICU have become available. Efforts to find the optimal timing for the initiation of RRT continue. The results of the recently published STARRT-AKI trial show no survival benefit to an early compared with delayed strategy for RRT initiation in patients without an urgent indication. ${ }^{7}$ In addition, once the decision to initiate RRT is made, there is emerging evidence showing the association of ultrafiltration rates that are too fast or too slow with mortality. ${ }^{8}$

Importantly, the AKI research community has developed a commitment to exploring the impact of this syndrome on patients once they leave the ICU. We have learned that a longer duration of $\mathrm{AKI}$ is associated with worse outcomes when compared with $\mathrm{AKI}$ that rapidly resolves. The KDIGO AKI Work Group first proposed a definition equivalent to chronic kidney disease occurring before 90 days, termed acute kidney disease. $^{2}$ More recently, the Acute Disease Quality Initiative expanded this by proposing AKI-based staging for patients with acute kidney disease arising from AKI. ${ }^{9}$ Understanding that we should not be reassured by a return of normal renal function by serum creatinine, a renewed interest in concepts like renal functional reserve has emerged.

This issue of Critical Care Clinics provides a comprehensive review of current advances in the identification, classification, and treatment approaches to AKI. We wish to thank the outstanding group of experts that contributed their work. For those caring for patients with AKI in the ICU, we hope that this issue will serve as a guide of the current evidence and a source of excitement for future areas of study in the field of AKI research.

Dana Y. Fuhrman, DO, MS

Department of Critical Care Medicine and

Pediatrics

UPMC Children's Hospital of Pittsburgh

The Center for Critical Care Nephrology 4401 Penn Avenue, Children's Hospital Drive

Faculty Pavilion, Suite 2000 Pittsburgh, PA 15224, USA

John A. Kellum, MD, MCCM Department of Critical Care Medicine The Center for Critical Care Nephrology 3347 Forbes Avenue, Suite 220 Pittsburgh, PA 15213, USA 


\section{REFERENCES}

1. Hoste EA, Bagshaw SM, Bellomo R, et al. Epidemiology of acute kidney injury in critically ill patients: the multinational AKI-EPI study. Intensive Care Med 2015; 41(8):1411-23.

2. KDIGO AKIWG. Kidney Disease: Improving Global Outcomes (KDIGO) clinical practice guideline for acute kidney injury. Kidney Int Suppl 2012;2(1):1-141.

3. Jetton JG, Boohaker LJ, Sethi SK, et al. Incidence and outcomes of neonatal acute kidney injury (AWAKEN): a multicentre, multinational, observational cohort study. Lancet Child Adolesc Health 2017;1(3):184-94.

4. Kaddourah A, Basu RK, Goldstein SL, et al, Assessment of Worldwide Acute Kidney Injury RAaEI. Oliguria and acute kidney injury in critically ill children: implications for diagnosis and outcomes. Pediatr Crit Care Med 2019;20(4):332-9.

5. Amathieu R, Al-Khafaji A, Sileanu FE, et al. Significance of oliguria in critically ill patients with chronic liver disease. Hepatology 2017;66(5):1592-600.

6. Post EH, Kellum JA, Bellomo R, Vincent JL. Renal perfusion in sepsis: from macroto microcirculation. Kidney Int 2017;91(1):45-60.

7. Bagshaw SM, Wald R, Adhikari NKJ, et al. Timing of initiation of renal-replacement therapy in acute kidney injury. N Engl J Med 2020;383(3):240-51.

8. Murugan R, Kerti SJ, Chang $\mathrm{CH}$, et al. Association of net ultrafiltration rate with mortality among critically ill adults with acute kidney injury receiving continuous venovenous hemodiafiltration: a secondary analysis of the Randomized Evaluation of Normal vs Augmented Level (RENAL) of Renal Replacement Therapy Trial. JAMA Netw Open 2019;2(6):e195418.

9. Chawla LS, Bellomo R, Bihorac A, et al. Acute kidney disease and renal recovery: consensus report of the Acute Disease Quality Initiative (ADQI) 16 Workgroup. Nat Rev Nephrol 2017;13(4):241-57. 